\title{
Cognitive Impairment: A Retrospective Study of Carotid Artery Stenosis
}

\section{Gennaro ${ }^{1^{*}}$}

${ }^{1}$ Department of Behavior and Brain Functions, Iran.

Corresponding Author: M Gennaro, Department of Behavior and Brain Function, Iran.

Received date: January 25, 2019; Accepted date: February 13, 2019; Published date: February 19, 2019.

Citation: M Gennaro. (2019). Cognitive Impairment: A Retrospective Study of Carotid Artery Stenosis. Brain and Neurological Disorders. 2(1); DOI: 10.31579/2642-973X/007

Copyright: @ 2019 M Gennaro. This is an open-access article distributed under the terms of The Creative Commons Attribution License, which permits unrestricted use, distribution, and reproduction in any medium, provided the original author and source are credited.

\section{Abstract \\ Acute ischemic stroke has been recognized as one key cause of vascular cognitive impairment (VCl). The purpose of this study was to evaluate the correlation between carotid artery stenosis and post $\mathrm{VCl}$ in acute ischemic stroke patients.}

Introduction

Number of studies have reported that cerebral vascular disease is associated with cognitive function and shows a key role in the decline of memory (Feliziani et al., 2010; Lehrner et al., 2005; Xu, Liu, Meyer, Yin, \& Zhang, 2007). It has been recognized that vascular cognitive impairment (VCI) is correlated with disability worldwide and is the most common manifestation of severe cerebrovascular disease (Hachinski, 2007; Kearney-Schwartz et al., 2009; Sachdev et al., 2014). Notably, stroke may happen with risk factors of vascular disease and cause decline of cognitive function (Dempsey, Vemuganti, Varghese, \& Hermann, 2010; Elias et al., 2004). VCI is one of the consequence of stroke affecting and has been found in $25 \%-75 \%$ stroke patients (Haring, 2002; Mlekusch et al., 2008; Zhou et al., 2009). These studies indicate that there are connections between cerebrovascular disease and post stroke VCI.

Accumulating evidence showed that patients with severe carotid artery stenosis are correlated with VCI in memory (Inzitari et al., 2000; Popovic et al., 2011; Sztriha, Nemeth, Sefcsik, \& Vecsei, 2009). Patients with high-grade carotid artery disease showed lower scores on cognitive function tests than normal controls (Sander et al., 2010; Silvestrini et al., 2011; Zhong et al., 2011, 2012). Haley et al. (2007) reported that carotid artery stenosis was associated with worse neuropsychological performance. Recent study showed that both symptomatic and asymptomatic carotid stenosis are associated with cognitive impairment (Wang, Mei, \& Zhang, 2016). In contrast, previous epidemiological reports showed the correlations between intima-media thickness of carotid and VCI in patients without stroke (Mathiesen et al., 2004; Pettigrew, Thomas, Howard, Veltkamp, \& Toole, 2000). Interestingly, recent report by Yue et al. (2016b) demonstrated that intima-media thickness of carotid was positively correlated with VCI in acute ischemic stroke patients and patients with cognitive impairment had a higher proportion of large artery stroke when compared to patients with good cognition. However, most articles reported outcomes within the first 6 months after stroke onset, and few studies investigated the correlations between the grade of carotid stenosis and 1 year post stroke VCI in patients with a major stroke.

\section{Materials and Methods}

\section{Study population}

In this study, 382 consecutive Chinese participants with first ever stroke were enrolled from January 2013 to December 2014 in Jining No.1 People's Hospital. The first ever stroke were defined based on the criteria of WHO (Aho et al., 1980). Imaging of brain was available for all patients. The inclusion criteria was: (1) patients age older than 18 years; (2) the diagnosed stroke was confirmed by magnetic resonance imaging (MRI) or computed tomography (CT); (3) less than 1 week from the first ever stroke; (4) the informed consent was provided by participant.
The criteria of exclusion was: (1) other diseases on brain, including tumors, vascular malformations, abscesses, or multiple sclerosis; (2) transient ischemic attack; (3) medical treatment during the study; (4) patients without carotid artery stenosis; (4) patients had previous cognitive impairment. This is a double blind retrospective study. This research was approved by the Ethics Committee of Jining No.1 People's Hospital and all study participants provided the informed written consent.

\section{Data collection}

Information regarding demographic data (alcohol use, age, education, sex, history of disease), vascular risk factors and past medical history were recorded via questionnaires at time of stroke onset. Intake $100 \mathrm{ml}$ of liquor per day three times 1 week for at least 1 year was defined as alcohol use. The physical activity both during leisure time and at work was evaluated. Previous history of diseases, including stroke, myocardial infarction, diabetes, hypertension, atrial fibrillation, coronary artery disease, and hypercholesterolemia were recorded via self-reporting. The past medical history of using antihypertensive, glucose lowering and cholesterol lowering medications $<2$ weeks prior to the baseline interview was also recorded through self-reporting.

\section{Ultrasound examination}

All participants were examined by carotid ultrasound during hospitalization. One experienced operator did all the measurements using B-mode ultrasound according to the Atherosclerosis Risk in Communities protocol (Li et al., 1994; Riley et al., 1991). The ultrasound measurement included scanning the left and right carotid arteries, bifurcations, and internal carotid arteries (the first $2 \mathrm{~cm}$ ). The density of plaques was also examined. The grade of carotid artery stenosis was classified in two groups according to previous report (Faught et al., 1994): low-grade stenosis $(<70 \%)$ and high-grade stenosis $(\geq 70 \%)$, according to peak systolic velocity of the internal carotid/common carotid artery.

\section{Follow-up and neuropsychological evaluation}

The cognitive function of the participants was measured using the Mini Mental State Examination (MMSE) by one investigator blinded to clinical characteristics of participants. The MMSE measurement includes orientation to place and time, calculation and attention, memory, and language. Higher score indicates better cognitive function. Cognitive dysfunction is considered as MMSE scores <24 (Yue et al.)

\section{Statistical analysis}

All statistical analysis was performed by Prism (GraphPad Software, Inc.). Simple descriptive statistics were calculated using means and standard deviations for continuous data and percentages for categorical data. The data dispersion was checked before analysis. Differences between groups were determined by using unpaired $t$ test (two tailed) and Chi square test or Fisher's exact test. Logistic regression analysis was performed for categorical-dependent variables. $p$ values of $<.05$ were regarded as statistically significant. 


\section{Results}

Of the 382 participants who were initially registered in this study, 17 patients were excluded as not meeting requirements during the followup period. The remaining participants $(n=365)$ were evaluated at 1 year later after stroke; neuropsychological assessment data and ultrasound images were obtained. Clinical characteristics of participants categorized by cognitive function status were summarized in Table 1. Among the 365 participants, 136 (37.3\%) were diagnosed with VCI, which was defined as an MMSE score <24, and 229 $(62.7 \%)$ participants were determined with good cognition. Older age $\left(p<.01, t=7.8, R^{2}=.14\right)$, lower education level $(p<.01)$, and history of atrial fibrillation $(p<.01)$ were correlated with decline of cognitive function. Moreover, participants with VCI exhibited a higher likelihood of high grade of carotid stenosis compared to good cognition $(25.7 \%$ vs. $14.4 \%, p<.01)$.

\begin{tabular}{|c|c|c|c|c|}
\hline & \begin{tabular}{|l|} 
Cognitively not impaired \\
$(M M S E \geq 24) n=229$
\end{tabular} & \begin{tabular}{|l|} 
Cognitively impaired \\
$(\mathrm{MMSE}<24) n=136$
\end{tabular} & $p$ value & Other statistical values \\
\hline Age $($ mean $\pm S D)$ & $61.3 \pm 11.8$ & $71.9 \pm 13.6$ & $<.01$ & $t=7.8, R^{2}=.14$ \\
\hline Male, $n(\%)$ & $114(49.8 \%)$ & $72(52.9 \%)$ & .67 & \\
\hline Married, $n(\%)$ & $218(95.2 \%)$ & $131(96.3 \%)$ & .70 & \\
\hline $\operatorname{BMI}\left(\mathrm{kg} / \mathrm{m}^{2}\right)$ & $23.6 \pm 4.8$ & $24.1 \pm 5.7$ & .37 & \\
\hline SBP, mm Hg & $143.2 \pm 21.5$ & $146.7 \pm 24.3$ & .15 & \\
\hline DBP, mm Hg & $87.1 \pm 13.4$ & $85.7 \pm 12.3$ & .32 & \\
\hline \multicolumn{5}{|l|}{ Lipid profile and glucose levels } \\
\hline $\mathrm{TC}, \mathrm{mmol} / \mathrm{L}$ & $4.8 \pm 1.4$ & $4.9 \pm 1.7$ & .54 & \\
\hline FBG, $\mathrm{mmol} / \mathrm{L}$ & $6.4 \pm 2.2$ & $6.7 \pm 2.5$ & .23 & \\
\hline $\mathrm{HDL}, \mathrm{mmol} / \mathrm{L}$ & $1.3 \pm 1.1$ & $1.5 \pm 1.2$ & .10 & \\
\hline $\mathrm{LDL}, \mathrm{mmol} / \mathrm{L}$ & $2.7 \pm 1.2$ & $2.9 \pm 1.3$ & .14 & \\
\hline Alcohol use (Yes) & $87(38.0 \%)$ & $55(40.4 \%)$ & .66 & \\
\hline Tobacco use (Yes) & $96(41.9 \%)$ & $50(36.8 \%)$ & .47 & \\
\hline \multicolumn{5}{|l|}{ Education level (years of schooling) } \\
\hline $0-5, n(\%)$ & $52(22.7 \%)$ & $81(59.6 \%)$ & $<.01$ & $\mathrm{OR}=0.19, \mathrm{CI}=0.10-0.36$ \\
\hline$\geq 6, n(\%)$ & $177(77.3 \%)$ & $55(40.4 \%)$ & & \\
\hline \multicolumn{5}{|l|}{ History of diseases, $n(\%)$} \\
\hline \begin{tabular}{|c|} 
Hypertension \\
\end{tabular} & $156(68.1 \%)$ & $96(70.6 \%)$ & .76 & \\
\hline Diabetes & $53(23.1 \%)$ & $36(26.5 \%)$ & .62 & \\
\hline Hypercholesterolemia & $71(31.0 \%)$ & $35(25.7 \%)$ & .34 & \\
\hline Atrial fibrillation & $32(14.0 \%)$ & $39(28.7 \%)$ & $<.01$ & \\
\hline Coronary artery disease & $14(6.1 \%)$ & $10(7.4 \%)$ & .77 & \\
\hline \multicolumn{5}{|l|}{ Stroke subtypes, $n(\%)$} \\
\hline Large artery stroke & $123(53.7 \%)$ & $97(71.3 \%)$ & $<.01$ & $\mathrm{OR}=0.45, \mathrm{CI}=0.25-0.82$ \\
\hline Supratentorial cardioembolic & $16(7.0 \%)$ & $13(9.6 \%)$ & .42 & \\
\hline $\begin{array}{l}\text { Supratentorial } \\
\text { atherothrombotic }\end{array}$ & $15(6.6 \%)$ & $10(7.4 \%)$ & .77 & \\
\hline \multicolumn{5}{|l|}{ Affected cerebral artery $n(\%)$} \\
\hline Middle cerebral artery & $96(41.9 \%)$ & $63(46.3 \%)$ & .47 & \\
\hline Anterior cerebral artery & $61(26.6 \%)$ & $41(30.1 \%)$ & .53 & \\
\hline Posterior cerebral artery & $72(31.4 \%)$ & $32(23.5 \%)$ & .20 & \\
\hline \multicolumn{5}{|l|}{ Degree of carotid stenosis } \\
\hline$<70 \%$ & $196(85.6 \%)$ & $35(25.7 \%)$ & $<.01$ & $\mathrm{OR}=18.0, \mathrm{CI}=8.7-37.1$ \\
\hline$\geq 70 \%$ & $33(14.4 \%)$ & $101(74.3 \%)$ & & \\
\hline
\end{tabular}

$S D$, standard deviation; MMSE, mini-mental state examination; SBP, systolic blood pressure; DBP, diastolic blood pressure; TC, total cholesterol; FBG, fasting blood glucose; HDL, high-density lipoprotein cholesterol; LDL, low-density lipoprotein cholesterol; BMI, body mass index; OR,

odds ratio; CI, 95\% confidence interval.

Similarly, compared with good cognitive, participants with VCI showed a higher proportion of large artery stroke $(p<.01)$, while no significant difference was found in cardioembolism stroke. The serum levels of TC, FBG, HDL, and LDL were also determined, however, no difference was observed between good cognitive and VCI groups. Further, there were no significant differences among other general characteristics of participants including gender, marital status, weight, blood pressure, and alcohol use, history of hypertension, diabetes, hypercholesterolemia, and coronary artery disease.

\section{Table 1}

The general characteristics of participants $(n=365)$ stratified by cognitive status 


\section{Discussion}

This study investigated the relationship between the grade of carotid stenosis and cognitive disfunction in ischemic stroke patients. Our results showed a difference in MMSE between patients with a stenosis grade of $<70 \%$ and patients with a stenosis grade of $>70 \%$, indicating that acute ischemic stroke patients who had higher grade carotid artery stenosis were at high likelihood of post stroke cognitive disfunction. The association between VCI and severe carotid stenosis persisted even after adjusting for potential confounders.

It has been well known that carotid artery stenosis disease was correlated with decline of cognitive function in patients without stroke (Cerhan et al., 1998). In addition, another study showed that increased carotid artery intima-media thickness was linked with cognitive disfunction in patients with atherosclerotic plaques (Auperin et al., 1996). In this study, we observed that older age, lower education level, and history of atrial fibrillation were correlated with decline of cognitive function. Similarly, compared with good cognitive, participants with VCI showed a higher proportion of large artery stroke. These results are consistent with previous report that highgrade carotid stenosis has been known as a predictor of VCI in a prospective study (Johnston et al., 2004). Talelli et al. (2004) also reported that the cognitive impairment of 1 year after stroke was independently correlated with higher common carotid artery intimamedia thickness. Interestingly, one recent study showed that cognitive disfunction was correlated with severe right carotid artery stenosis (Yue et al., 2016a). In this study, we also found that severe carotid stenosis was positive associated with VCI. We showed a difference in MMSE between patients with a stenosis grade of $<70 \%$ and patients with a stenosis grade of $>70 \%$. These results suggest that severe carotid stenosis have important effect on VCI. Additionally, it has been demonstrated that cerebral hypoperfusion caused by severe carotid stenosis was correlated with decline of cognitive function (Consoli, Pasi, \& Pantoni, 2012). Thus, these findings highlight the potential value of severe carotid stenosis as a potential predictor of VCI in patients with acute stroke.

\section{References}

1. Aho K, Harmsen P, Hatano S, Marquardsen J, and Smirnov V. E, et al, (1980). Cerebrovascular disease in the community: Results of a who collaborative study. Bulletin of the World Health Organization, 58, 113-130.

2. Auperin A, Berr C, Bonithon-Kopp C, Touboul P. J, and Ruelland I, et al, (1996). Ultrasonographic assessment of carotid wall characteristics and cognitive functions in a community sample of 59- to 71-year-olds. The eva study group. Stroke, 27, 1290-1295.
Casey J. E, Ferguson G. G, Kimura D, \& Hachinski V. C. (1989). Neuropsychological improvement versus practice effect following unilateral carotid endarterectomy in patients without stroke. Journal of Clinical and Experimental Neuropsychology, 11, 461470.

4. Cerhan J. R, Folsom A. R, Mortimer J. A, Shahar E, and Knopman D. S, et al, (1998). Correlates of cognitive function in middle-aged adults. Atherosclerosis risk in communities (aric) study investigators. Gerontology, 44, 95-105.

5. Consoli A, Pasi M, \& Pantoni L. (2012). Vascular mild cognitive impairment: Concept, definition, and directions for future studies. Aging Clinical and Experimental Research, 24, 113-116.

6. Dempsey R. J, Vemuganti R, Varghese T, \& Hermann B. P. (2010). A review of carotid atherosclerosis and vascular cognitive decline: A new understanding of the keys to symptomology. Neurosurgery, 67, 484-493; discussion 493-484.

7. Desmond D. W, Moroney J. T, Paik M. C, Sano M, and Mohr J. P., et al, (2000). Frequency and clinical determinants of dementia after ischemic stroke. Neurology, 54, 1124-1131.

8. Elias M. F, Sullivan L. M, D'Agostino R. B., Elias P. K, and Beiser A, et al, (2004). Framingham stroke risk profile and lowered cognitive performance. Stroke, 35, 404-409.

9. Elwood P. C, Pickering J, Bayer A, \& Gallacher J. E. (2002). Vascular disease and cognitive function in older men in the caerphilly cohort. Age and Ageing, 31, 43-48.

10. Faught W. E, Mattos M. A, van Bemmelen P. S, Hodgson K. J, and Barkmeier L. et al, (1994). Color-flow duplex scanning of carotid arteries: New velocity criteria based on receiver operator characteristic analysis for threshold stenoses used in the symptomatic and asymptomatic carotid trials. Journal of Vascular Surgery, 19, 818-827; discussion 827-818.

11. Feliziani F. T, Polidori M. C, De Rango P, Mangialasche F, and Monastero R, et al, (2010). Cognitive performance in elderly patients undergoing carotid endarterectomy or carotid artery stenting: A twelve-month follow-up study. Cerebrovascular Diseases, 30, 244-251.

12. Fisher M. (1951). Senile dementia- a new explanation of its causation. Canadian Medical Association Journal, 65, 1-7.

13. Greiffenstein M. F, Brinkman S, Jacobs L, \& Braun P. (1988). Neuropsychological improvement following endarterectomy as a function of outcome measure and reconstructed vessel. Cortex, 24, 223-230. 